\title{
Simulación de trazos de rayos de un triplete de Cooke
}

\author{
Simulation of ray tracing Cooke triplet \\ R.A.Montalvo* ${ }^{*}$, J.I Choque ${ }^{2}$ \\ ${ }^{1}$ Facultad de Ciencias Físicas, Universidad Nacional Mayor de San Marcos, Lima, Perú \\ ${ }^{2}$ Centro de Investigaciones en Óptica, Guanajuato, México
}

Recibida en 04 de Agosto, 2019. Revisado en 30 de Septiembre, 2019. Aceptado en 11 de Noviembre, 2019.

\begin{abstract}
En el presente trabajo, desarrollamos el modelo teórico de un triplete de Cooke en un ambiente que ejecuta programas con rapidez; lo que significa que puede construir superficies; incrementándolas y resolviendo diseños más complejos y elaborados. Este resultado es comparado con el dispositivo óptico elaborado con el software OSLO EDU [1]. Mostramos como un simple código se presenta como una herramienta computacional para la enseñanza de la óptica geométrica y paraxial de primer orden para un curso introductorio de óptica.
\end{abstract}

Palabras clave: Diseño óptico, triplete de Cooke, óptica geométrica paraxial

In this paper, we developed the theoretical model Cooke triplet in an environment that runs programs quickly; which means you can build surfaces; increasing them and solving more complex and elaborated designs. This result is compared with the optical device developed with OSLO EDU [1] software. We show how a simple code is presented as a computational tool for the teaching of geometric and paraxial optics of the first order for an introductory optics course.

Keywords: Optical design, Cooke triplet, geometric paraxial optics

\section{Introducción}

Existen softwares de diseño óptico en el mercado asistidos por computadora que se utilizan para modelar, analizar, optimizar y brindar soporte de fabricación para el desarrollo de sistemas ópticos y sus diversas aplicaciones; CODE V [2], ZEMAX [3], OSLO EDU entre otros, realizan diseños en un ambiente gráfico interactivo con el uso elementos ópticos tales como espejos, lentes, prismas, etc; que permiten visualizar el desarrollo del detalle de la óptica geométrica paraxial aplicada a los parámetros de cada elemento que se va incorporando sucesivamente, hasta obtener finalmente, los resultados esperados del sistema óptico que se desea construir. El diseño de lentes solía ser una habilidad reservada para unos pocos profesionales quienes empleaban softwares de análisis y diseño óptico, propiedad de empresas que poseían grandes y costosas computadoras. Hoy en día softwares fácilmente disponibles son las herramientas de diseño de lentes accesibles a la comunidad de ingeniería óptica; siendo un componente importante para los estudiantes que se forman en esta área. En la actualidad programas como CODE V, ZEMAX, OSLO EDU versión 6.4.2, entre otros son utilizados y aceptados como una herramienta estándar por compañías de todo el mundo que lo utilizan para crear mejores diseños a menor costo y fabricarlos de manera más eficiente. CODE V fue lanzado al mercado en 1975; sus líneas de comandos extremadamente eficientes y sus

*Correo electrónico: rmontalvob@unmsm.edu.pe. algoritmos de análisis son reconocidos por su precisión y velocidad produciendo modelamientos que brindan exactitud en los diseños experimentales. A mediados de 1990, ZEMAX comenzó a ofrecer su software y servicios ópticos para ayudar a los investigadores; dentro de sus herramientas de prototipos virtuales, incluye OpticStudio®, que posee herramientas y asistentes fáciles de aprender brindando una simulación y eficiencia para diseños de cualquier sistema óptico. Desde 1992, Lambda Research Corporation ha desarrollado el programa OSLO; fue el primer software que demostró que el diseño de una serie de lentes ópticas podía llevarse a cabo utilizando computadoras de escritorio. Este software está disponible en tres ediciones; Premium, Standard y Light. Todos estos programas incluyen varios catálogos de vidrios y lentes en existencia en sus bases de datos y trabajan con una interface de usuario personalizable basada en Windows. Funcionan mediante el trazado de rayos, simulando la propagación de estos a través de un sistema óptico. Pueden modelar el efecto de elementos ópticos como lentes simples, asféricas, espejos, cálculo del rendimiento óptico etc, ahorrarando en costos de fabricación y brindando resultados confiables en menos tiempo; pese a todos estos beneficios y facilidades su unica desventaja se muestra en su precio que oscila entre los 4000 y 20000 dolares.

En 1893 el primer sistema de lentes que permitía eliminar la mayor parte de la distorsión óptica o aberración en la zona exterior de las lentes fue creado. Conocido como triplete Cooke fue inventado por H. Dennis Taylor 
usando los avances de la teoría de Seidel 4], y patentado en 1895. El triplete Cooke es un objetivo muy interesante para la aplicación en el diseño de lentes porque combina la simplicidad de la construcción (consta de solo tres lentes espaciados entre si; un elemento de vidrio negativo bicóncavo en el centro y un elemento convexo a cada lado) y la facilidad de una corrección completa de las aberraciones. El conjunto posee catorce variables disponibles; dos espaciamientos entre las tres lentes, las curvaturas de las seis superficies, tres tipos de vidrios y tres espesores diferentes; la modificación de estos parámetros hacen posible la eliminación de las aberraciones de Seidel. Un triplete de Cooke es una lente acromática, cada uno de los dos elementos positivos deben estar hecho de un vidrio tipo crown [5] y el elemento negativo un vidrio tipo flint [6]. Por razones prácticas y de eficiencia, los elementos positivos generalmente están hechos del mismo tipo de vidrio. Las distancias entre las lentes en un triplete de Cooke son párametros importantes para el control de dispersión de los vidrios crown y flint (baja y alta dispersión respectivamente). Si la dispersión es mínima, los elementos de la lente se solaparían; caso contrario se expandería excesivamente. La diferencia de los índices de refracción de las lentes también es importante en la solución óptica. Los índices de las lentes positivas deben ser ligeramente menores al de la lente negativa. La suma de todas las curvaturas producidas por los índices de refracción deben aproximarse a cero, de esta forma el campo de foco sería plano (curvatura de campo de Petzval cero).

En la Figura 1 se aprecia la aplicacion de este sistema óptico; a la izquierda el diseño de un triplete Cooke, en la parte superior derecha la formación de la imagen sin aberraciones con el uso del triplete y en la inferior la formación de la imagen con una sola lente que produce imágenes con aberraciones.

En el presente trabajo se compara la información proporcionada por el código que hemos desarrollado con el resultado del programa Optics Software for Layout and Optimization, OSLO EDU. El software utiliza la óptica geométrica y paraxial en una interface gráfica para simular sistemas ópticos. OSLO EDU es una edición gratuita y esta disponible en su pagina web; está destinado para fines educativos con la única limitación que solo permite el modelamiento hasta un máximo de diez superficies ópticas. En nuestro caso el diseño elaborado por nues-
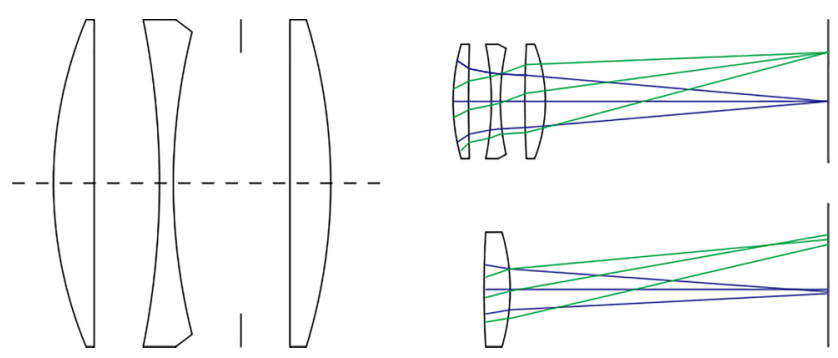

Figura 1: Formación de imagen con el triplete Cooke. tro código será contrastado con el software OSLO EDU; durante la elaboración de un elemento óptico existe la posibilidad de introducir factores que reduzcan la calidad en la formación de la imagen final, esto debido a que los rayos no son paraxiales o la luz no es monocromática; el codigo elaborado en el presente trabajo esta libre de tales factores.

\section{Diseño de las superficies del triplete}

El detalle teórico usado en la elaboración del código se describe en el libro de Smith 7]. Los rayos, perpendiculares a los frentes de onda, se representan por rayos meridionales que cruzan el eje óptico y los oblícuos que contribuyen al tamaño y posición de la imagen. Luego que los rayos han atravesado un sistema óptico que puede estar compuesto por elementos reflectores y refractores, se consigue la formación final de la imagen de un objeto, despreciando los efectos de difracción.

Las características de la imagen resultante de un sistema óptico puede ser facilmente calculado con el conocimiento de la ubicación de algunos puntos en el eje óptico; los puntos focales y los puntos principales son algunos de ellos. Los puntos focales son aquellos en los cuales los rayos de luz provenientes desde un punto objeto axial infinitamente distante y paralelo al eje óptico coinciden en un punto común del eje.

Los rayos ingresan al sistema y emergen extendiéndose hasta cruzarse; estos puntos de intersección definirán una superficie, generalmente conocida como el plano principal. En un sistema óptico bien corregido, las superficies principales son curvas centradas respecto al eje. La intersección de superficies con el eje son puntos principales (primer punto principal $p p p$, segundo punto principal $s p p$ ) y el punto donde convergen los rayos es el foco o punto focal (primer punto focal $p p f$ y segundo punto focal $s p f$ ). De lo anterior, se define como longitud focal efectiva $e f l$ a la distancia de un plano principal al segundo punto focal, longitud focal posterior $b f l$ o foco a la distancia de la última superficie del sistema al segundo punto focal, y la longitud frontal efectiva $f f l$ a la distancia de la primera superficie respecto al primer punto focal, Figura 2. Nuestro sistema óptico es axialmente simétrico y se encuentra en contacto con el aire.

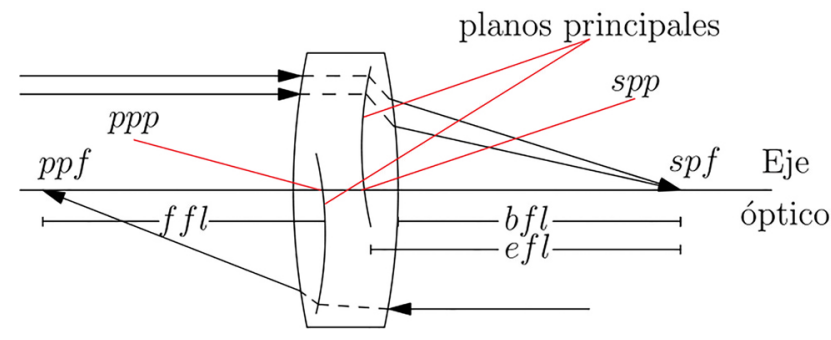

Figura 2: Localización de los puntos principales y focales de de un sistema óptico general. 
En la región paraxial donde las distancias de los rayos desde los ejes son infinitesimales respecto a la distancia del objeto y la imagen, las superficies se pueden tratar como si fueran planos, de ahí el nombre; "planos" principales, Figura 3. Cuando los puntos cardinales coinciden con los planos principales, $F_{1}$ y $F_{2}$ son los focos y $P_{1}$ y $P_{2}$ los puntos principales, el rayo $\overline{O B}$ es paralelo al eje óptico y atraviesa el foco $F_{2}$ luego de refractarse por el segundo plano principal del sistema óptico, el rayo $\overline{O F_{1} C}$ cruza el primer punto focal $F_{1}$ y emerge paralelo al eje en el punto $O^{\prime}$, la interseccion de estos dos puntos en $O^{\prime}$ ubica la imagen del objeto de $O$, siendo la magnificación transversal $m=h^{\prime} / h$

Para calcular la propagación de los rayos a través de los sistemas ópticos, se adopta una serie de convenciones sobre la nomenclatura y los signos de las variables. Consideramos el sistema óptico de la Figura 4 que muestra el rayo de luz incidente $\overline{G Q}$ sobre una superficie refractora esférica, la proyección $\overline{G Q P}$ considera el haz incidente sin refracción cruzando el eje óptico a una distancia $L$ de la superficie esférica en el punto $O$. El rayo $\overline{G Q}$ es refractado por la superficie y cruza el eje óptico en $P^{\prime}$, a una distancia $L^{\prime}$ de la superficie. La superficie tiene un radio $R$ con centro de curvatura en $C$ y separa los dos medios de índice de refracción $n$ en el lado izquierdo y $n^{\prime}$ en el derecho. La proyección de la línea $\overline{G Q P}$ hace un (ángulo $\equiv \angle) \angle U$ con el eje óptico para el caso sin refracción, y

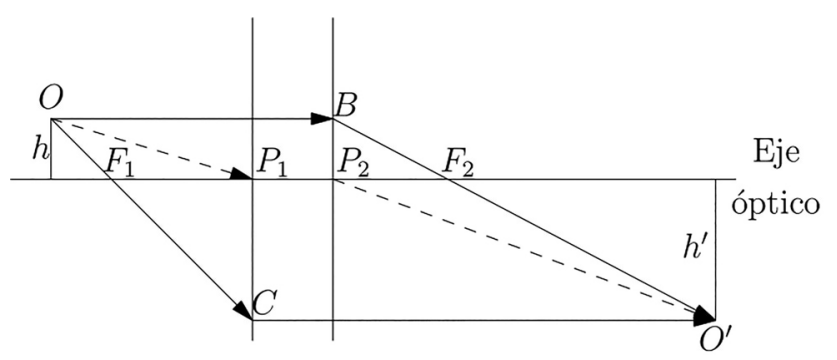

Figura 3: Traza de rayos a través de los puntos pricipales como los planos y distancias focales.

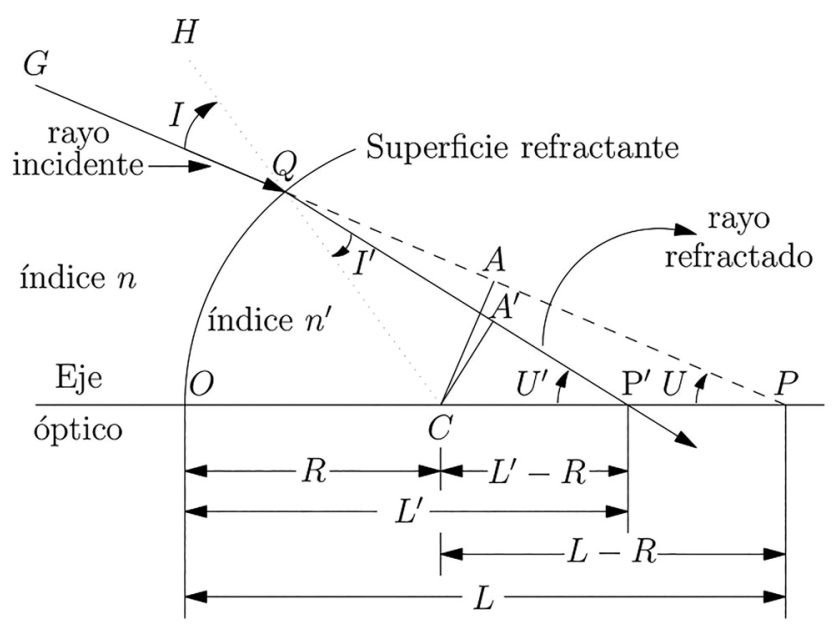

Figura 4: Refracción de la luz por una superficie esférica. el rayo $\overline{Q P^{\prime}}$, un $\angle U^{\prime}$ considerando la refracción; $\angle I$ es el ángulo entre el rayo incidente más su proyección lineal $\overline{G Q P}$ y la normal $\overline{H Q C}$ a la superficie refractante que se intersectan en el punto $Q$, y el $\angle I^{\prime}$ está formado entre el rayo refractado $\overline{Q P^{\prime}}$ y la normal $\overline{H Q C}$.

Las convenciones de signos que se observan son: 7

a) El radio es positivo si el centro de curvatura está a la derecha de la superficie.

b) Las distancias a la derecha de la superficie son positivas, a la izquierda, negativas.

c) Los ángulos de incidencia $\angle I$ y refracción $\angle I^{\prime}$ son positivos si el rayo es rotado en el sentido horario para alcanzar la normal.

d) Los ángulos pendientes $\angle U$ y $\angle U^{\prime}$ son positivos si el rayo es rotado en el sentido antihorario para alcanzar al eje óptico.

e) La luz viaja de izquierda a derecha.

Un conjunto de ecuaciones se derivan de la Figura 4. por ejemplo en el (triángulo $\equiv \triangle) \triangle P A C$ tenemos que

$$
\overline{C A}=(L-R) \operatorname{sen}(-U)=(R-L) \operatorname{sen} U,
$$

en el $\triangle P^{\prime} A C$ se determina que

$$
\overline{C A^{\prime}}=\left(L^{\prime}-R\right) \operatorname{sen}\left(-U^{\prime}\right),
$$

en el $\triangle Q A C$ observamos que

$$
\operatorname{sen} I=\frac{\overline{C A}}{R},
$$

en el $\triangle Q A^{\prime} C$ se deduce que

$$
\operatorname{sen} I^{\prime}=\frac{\overline{C A^{\prime}}}{R},
$$

y angularmente se consideran las siguientes relaciones, comenzando en el $\triangle P Q C$, el ángulo exterior $\angle Q C O \equiv$ $-\angle U+\angle I$, para el $\triangle P^{\prime} Q C$ el ángulo exterior $\angle Q C O \equiv$ $-\angle U^{\prime}+\angle I^{\prime}$ y de la igualdad de ambos resultados obtenemos que

$$
\angle U^{\prime}=\angle U-\angle I+\angle I^{\prime}
$$

De las relaciones citadas en el párrafo anterior y de la ley de Snell, obtenemos

$$
\overline{C A^{\prime}}=\frac{n}{n^{\prime}} \overline{C A}
$$

donde $n$ y $n^{\prime}$ son los índices de refracción en los medios de la izquierda y la derecha, respectivamente.

En la óptica geométrica se suele usar la aproximación paraxial que consiste en la aproximación de ángulos pequeños, especialmente cuando los rayos de luz van próximos a los ejes ópticos y producen ángulos pequeños con los mismos. A estos rayos se les denominan rayos paraxiales. Si $\theta$ es el ángulo pequeño entre el rayo incidente paraxial y el eje óptico, entonces en primera aproximación tenemos que $\operatorname{sen} \theta \approx \theta, \tan \theta \approx \theta \mathrm{y} \cos \theta \approx 1$. De la 
misma forma el ángulo de refracción es también pequeño. Para discriminar las ecuaciones de la óptica paraxial de la óptica geométrica usamos las letras minúsculas para la notación paraxial [7], exceptuando el radio de curvatura de la superficie óptica, para ello reescribimos las ecuaciones anteriores basados en la Figura 4 como

$$
\begin{aligned}
\overline{c a} & =(R-l) u, \\
\overline{c a^{\prime}} & =\left(R-l^{\prime}\right) u^{\prime} \Rightarrow l^{\prime}=R-\frac{\overline{c a^{\prime}}}{u^{\prime}}, \\
i & =\frac{\overline{c a}}{R}, \\
i^{\prime} & =\frac{n}{n^{\prime}} i \\
u^{\prime} & =u-i+i^{\prime} \\
\overline{c a^{\prime}} & =\frac{n}{n^{\prime}} \overline{c a} .
\end{aligned}
$$

Expresando los ángulos en radianes, reemplazamos la Ec. (7) en la Ec. 9) y la ecuación resultante en la Ec. 10. Las últimas dos ecuaciones obtenidas son reemplazadas en la Ec. 111 y representadas en la Ec. 13.

$$
\frac{u^{\prime}}{u}=\frac{l}{R}+\frac{n}{n^{\prime}} \frac{(R-l)}{R} .
$$

De la misma forma, reemplazamos las Ecs. (7) y(8) en la Ec.12 obteniéndose

$$
\frac{u^{\prime}}{u}=\frac{n}{n^{\prime}} \frac{(R-l)}{\left(R-l^{\prime}\right)} .
$$

Igualando estas dos últimas ecuaciones (13) y (14), y realizando un poco de algebra llegamos a

$$
\frac{n^{\prime}}{l^{\prime}}=\frac{\left(n^{\prime}-n\right)}{R}+\frac{n}{l},
$$

que relaciona las distancias $l$ y $l^{\prime}$ de los puntos de intersección entre los haces no refractado y refractado con el eje óptico, respectivamente, los índices de refracción y el radio de curvatura. Esta aproximación se describe como el dominio de primer orden. La Ec. (15) suele ser conocida como la la ecuación del fabricante de lente.

Considerando que el haz incide sobre la superficie a una altura $h$ respecto al eje óptico entonces los ángulos $u$ y $u^{\prime}$, Figura 5, están dados como $u=h /-l$ y $u^{\prime}=h /-l^{\prime}$, las cuales reemplazamos en la Ec. 15 obteniéndose el parámetro denominado apertura numérica de la imagen

$$
n^{\prime} u^{\prime}=n u-h \frac{n^{\prime}-n}{R},
$$

donde $n u$ es la apertura numérica del objeto en la aproximación paraxial y $1 / R$ la potencia del elemento óptico.

En general, los rayos paraxiales atraviesan más de una superficie refractora, comenzamos analizando el paso de un rayo a través de dos superficies que define un medio óptico, Figura 6 .

El rayo incide en la primera superficie a una altura $h_{1}$ respecto al eje óptico y se refracta a través de la segunda

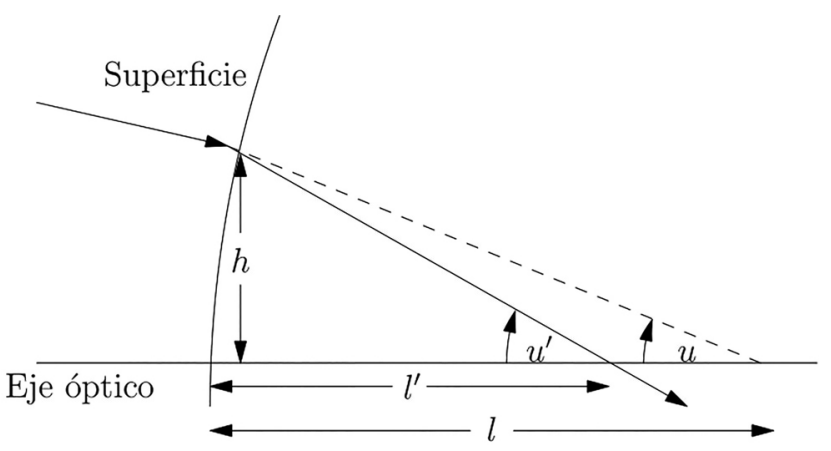

Figura 5: Rayo paraxial atravesando una superficie.

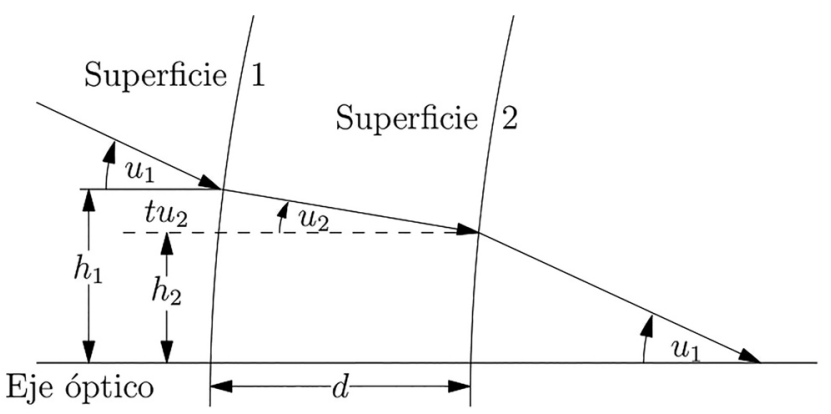

Figura 6: Rayo paraxial atravesando dos superficies separadas por una distancia d.

superficie hasta una altura $h_{2}$ respecto al mismo eje. La distancia entre las superficies a lo largo del eje óptico es $d$ y el ángulo de incidencia en el medio óptico sobre la segunda superfice es $u_{2}=u_{1}^{\prime}$ de modo que este haz refractado hace un ángulo $u_{2}^{\prime}$ con el eje óptico. La altura entre el haz incidente y el lado subtendido por el ángulo $u_{1}^{\prime}$ es $d u_{1}^{\prime}$, luego las alturas, considerando la convención de signos, están relacionadas por la ecuación

$$
h_{2}=h_{1}+d \frac{n_{1}^{\prime} u_{1}^{\prime}}{n_{1}^{\prime}},
$$

y las aperturas numéricas de las superficies debido a la refracción son

$$
\begin{aligned}
& n_{1}^{\prime} u_{1}^{\prime}=n_{1} u_{1}-h_{1} \frac{\left(n_{1}^{\prime}-n_{1}\right)}{R_{1}} \quad \mathrm{y} \\
& n_{2}^{\prime} u_{2}^{\prime}=n_{2} u_{2}-h_{2} \frac{\left(n_{2}^{\prime}-n_{2}\right)}{R_{2}},
\end{aligned}
$$

donde se observa que $n_{1} u_{1}=n_{2}^{\prime} u_{2}^{\prime}$ si consideramos que el medio que rodea al sistema óptico es el mismo, esto indica que $n_{1}=n_{2}^{\prime}$, luego $u_{1}=u_{2}^{\prime}$ y por otro lado, $n_{1}^{\prime} u_{1}^{\prime}=n_{2} u_{2}$ y $n_{1}^{\prime}=n_{2}$ porque el haz refractado en la primera superficie es el haz incidente en la segunda superficie; asi la apertura numérica está dada por

$$
n_{2}^{\prime} u_{2}^{\prime}=n_{1}^{\prime} u_{1}^{\prime}-h_{2} \frac{\left(n_{2}^{\prime}-n_{1}^{\prime}\right)}{R_{2}} .
$$

Cabe notar que reemplazando la Ec. (18) en la Ec. 20 iniciamos un proceso recursivo, que para una superficie $i$, 
las aperturas numéricas de las imágenes formadas serán

$$
n_{i}^{\prime} u_{i}^{\prime}=n_{i-1}^{\prime} u_{i-1}^{\prime}-\frac{h_{i}\left(n_{i}^{\prime}-n_{i-1}^{\prime}\right)}{R_{i}}
$$

y las alturas recursivamente son

$$
\begin{gathered}
h_{i}=h_{i-1}+d_{i-1} \frac{n_{i-1}^{\prime} u_{i-1}^{\prime}}{n_{i-1}^{\prime}} . \\
h_{i}=h_{i-1}+d_{i-1} u_{i-1}^{\prime} .
\end{gathered}
$$

Con las ecuaciones Ec. 21) y Ec. 23 podemos conocer la apertura numérica, la posición y el tamaño de la imagen formada por un sistema óptico completo. Hay que tener en cuenta que las alturas de los rayos paraxiales y las pendientes de los rayos son proporcionales (es decir, pueden multiplicarse por el mismo factor). El resultado de la escala se genera con los datos de otro rayo (que tiene la misma intersección axial).

\section{Resultados}

El proceso de diseño de un triplete Cooke que se describe en esta sección no es el que se usa en la actualidad. Este tipo de lente es especialmente interesante porque hay suficientes grados de libertad disponibles que permiten al diseñador la corrección de todas las aberraciones monocromáticas primarias, dos cromáticas y su potencia. El triplete consta de seis superfices, si a esto se agregan los índices de refracción y las dispersiones, entonces es posible cierta flexibilidad adicional en su diseño; especialmente si se debe controlar algun parámetro adicional, como la longitud focal posterior. Los parámetros usados para el diseño se muestran en la Tabla $1 ; R_{i}$ es el radio de curvatura, $d_{i}$ el espacio entre las superficies y $n_{i}$ el índice de refracción.

Para iniciar la traza de rayos, necesitamos indicar los valores iniciales $h$ y $u$ en la primera superficie para luego aplicar la ecuación de refracción para todas las superficies que se trabajen. En ocasiones, el objeto se asume como la primera superficie, en cuyo caso comenzamos aplicando la ecuación de transferencia entre el objeto y la siguiente superficie. Mostramos que todas las propiedades gaussianas del sistema se establecen mediante el trazado de al menos dos rayos; utilizando un único rayo, la ubicación de la imagen y su ampliación son hallados.

Tabla 1: Se muestran los datos de entrada para el diseño del triplete Cooke utilizados en la simulación.

\begin{tabular}{lcccc}
\hline Superf. (i) & $R_{i}(\mathrm{~mm})$ & $d_{i}(\mathrm{~mm})$ & $n_{i}$ & material \\
\hline 1 & 40,10 & 6,0 & 1,61272 & SK4 6] \\
2 & $-537,00$ & 10,0 & 1,0 & aire \\
3 & $-47,00$ & 1,0 & 1,62096 & FN11 \\
4 & 40,00 & 10,8 & 1,0 & aire \\
5 & 234,50 & 6,0 & 1,61272 & SK4 6$]$ \\
6 & $-37,90$ & $f_{b f l}$ & 1,0 & aire \\
\hline
\end{tabular}

Un segundo rayo nos brinda información sobre el tamaño proporcionado por las lentes individualmente. Aunque esta información no es necesaria, si es relevante en una situación práctica.

El primer paso es tabular los parámetros del problema con los signos apropiados asociados, siguiendo la convención de signos dada anteriormente. Los datos de entrada son las curvaturas de las superficies, las separaciones entre ellas y los índices de refracción; se encuentran en la Tabla 1.

Se especifica un valor arbitrario $h_{1}(\mathrm{~mm})$ para la primera superficie ya que algunos párametros como la longitud focal efectiva $e f l$, la longitud focal posterior $b f l$, la amplificación transversal $m$ y la posición del plano principal $\delta$ se representan como el cociente de cantidades paraxiales, cancelándose el valor de $h_{1}$.

El código fue realizado en MATLAB R2018a [8], los valores iniciales son $h_{1}=16,7(\mathrm{~mm})$ y $u_{1}=0$, la Tabla 2 muestra los valores de $h_{1}$ y $u_{1}^{\prime}$ para cada una de las seis superficies. Adicionalmente la longitud focal efectiva, la longitud focal posterior, la amplificación transversal y la posición del plano principal.

En la Figura 7, mostramos la simulación del triplete de Cooke realizado con el código desarrollado en MATLAB, el ángulo de ingreso a la primera superficie es cero; los datos ingresados al código se encuentran en la Tabla 1: los radios de curvatura, las distancias entre las superficies, sus índices de refracción con sus correspondientes materiales. En nuestro trabajo hemos introducido la información del objeto como los datos correspondientes a la primera superficie. De la Tabla 2 se obtienen los ángulos de ingreso de los rayos refractados y las alturas de las imágenes para cada elemento. Se aprecian las seis

Tabla 2: Ángulo refractado y tamaño de la imagen producida en cada superficie para $u_{1}=0$.

\begin{tabular}{ccc}
\hline Superficie & $u_{i}^{\prime}$ & $h_{i}(\mathrm{~mm})$ \\
\hline 1 & $-0,15823$ & 16,700 \\
2 & $-0,27314$ & 15,751 \\
3 & $-0,062392$ & 13,019 \\
4 & 0,100001 & 12,957 \\
5 & 0,039269 & 14,037 \\
6 & $-0,16741$ & 14,272 \\
\hline Longitud focal efectiva $e f l=99,7548 \mathrm{~mm}$ \\
Longitud focal posterior $b f l=85,2544 \mathrm{~mm}$ \\
Amplificación transversal $m=0,8546$ \\
Osición del plano principal $\delta=-14,5003 \mathrm{~mm}$
\end{tabular}

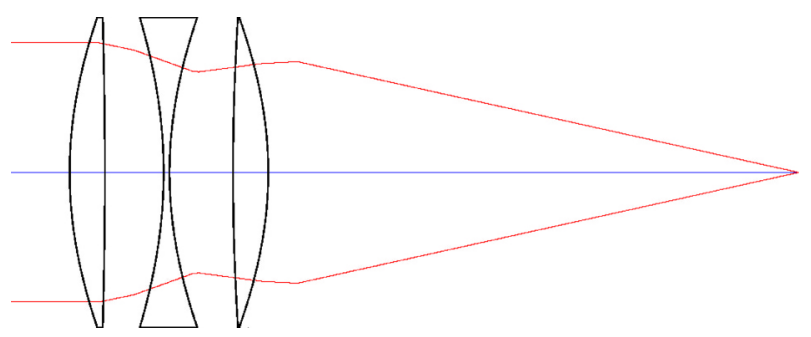

Figura 7: Diseño del triplete de Cooke realizado por MATLAB para $u_{1}=0$, con el uso de dos haces marginales 
superficies y dos haces marginales que convergen, para este sistema óptico, si se aumentase la incidencia de rayos, estos siempre convergerán en un único punto. El código del programa define la potencia de las superficies, calcula los ángulos de incidencia y refractados para determinar las longitudes focales y grafica el trazo de los rayos.

Para un segundo caso el ángulo de incidencia es diferente de cero, $u_{1}=\arcsin \left(\frac{h_{1}}{\sqrt{h_{1}^{2}+l_{1}^{2}}}\right)$, con condiciones iniciales $h_{1}=16,7 \mathrm{~mm}, l_{1}=-200 \mathrm{~mm}$, la simulación con MATLAB, los ángulos refractados y las alturas de las superficies se observan en la Figura 8 y la Tabla 3 respectivamente.

En la Figura 8 las alturas de las imágenes y los ángulos refractados, formadas en las superficies se incrementan, respecto al caso $u_{i}=0$. Como consecuencia de las Ec.(21) y Ec. 23, las longitudes focales efectiva y posterior, la posición de la imagen y su amplicación tambien lo hacen.

Finalmente en la región paraxial de primer orden las trayectorias de los rayos forman ángulos muy pequeños con el eje óptico por lo que el ángulo se aproxima a sus funciones seno y tangente y el coseno a 1; asi el ángulo de incidencia se expresa en función de la pendiente $u_{1}=\frac{h_{1}}{l_{1}}$

Ambas Figuras 8 y 9 son equivalentes, de las Tablas 3 y 4 vemos que los ángulos $u_{1}^{\prime}$ difieren en el orden de las diezmilésimas y las alturas de las imágenes de milésimas, esto debido a que los ángulos al ser muy pequeños, el arco de la superficie curva se aproxima a un segmento plano: $u_{1}=\arcsin \left(\frac{h_{1}}{\sqrt{h_{1}^{2}+l_{1}^{2}}}\right)=\frac{h_{1}}{l_{1}}$.

La información de la tabla 1 se introdujo al programa OSLO EDU para $u_{1}=0$, el programa proporciona la

Tabla 3: Ángulo refractado y tamaño de la imagen producida en cada superficie si la incidencia es $u_{1}=\arcsin \left(\frac{h_{1}}{\sqrt{h_{1}^{2}+l_{1}^{2}}}\right)$

\begin{tabular}{ccc}
\hline Superficie & $u_{i}^{\prime}$ & $h_{i}(\mathrm{~mm})$ \\
\hline 1 & $-0,10657$ & 16,700 \\
2 & $-0,19019$ & 16,061 \\
3 & $-0,0019297$ & 14,159 \\
4 & 0,21664 & 14,157 \\
5 & 0,10761 & 16,496 \\
6 & $-0,10359$ & 17,142 \\
\hline \multicolumn{2}{l}{ Longitud focal efectiva efl $=161,2060 \mathrm{~mm}$}
\end{tabular}

Longitud focal posterior $b f l=165,4737 \mathrm{~mm}$ Amplificación transversal $m=1,0265$

Posición del plano principal $\delta=4,2677 \mathrm{~mm}$

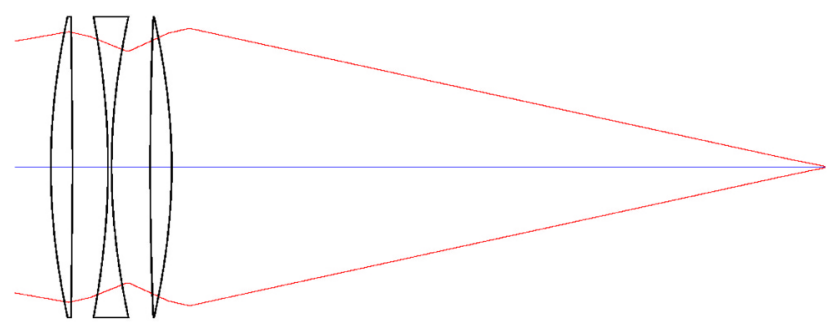

Figura 8: Diseño del triplete de Cooke realizado para $u_{1}=$ $\arcsin \left(\frac{h_{1}}{\sqrt{h_{1}^{2}+l_{1}^{2}}}\right)$
Tabla 4: Ángulo refractado y tamaño de la imagen producida en cada superficie para $u_{1}=\frac{h_{1}}{l_{1}}$.

\begin{tabular}{lcc}
\hline Superficie & $u_{i}^{\prime}$ & $h_{i}(\mathrm{~mm})$ \\
\hline 1 & $-0,10645$ & 16,700 \\
2 & $-0,19000$ & 16,061 \\
3 & $-0,0017894$ & 14,161 \\
4 & 0,21691 & 14,160 \\
5 & 0,10776 & 16,502 \\
6 & $-0,10345$ & 17,149 \\
\hline ongitud focal efectiva efl $=161,4367 \mathrm{~mm}$ \\
ongitud focal posterior $b f l=165,7749 \mathrm{~mm}$ \\
Amplificación transversal $m=1,0269$ \\
Posición del plano principal $\delta=4,3381 \mathrm{~mm}$
\end{tabular}

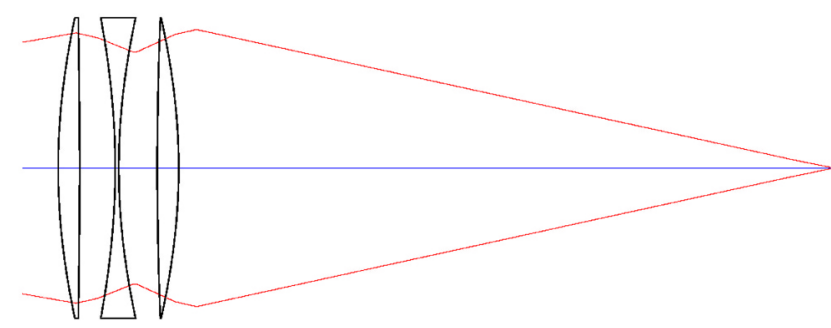

Figura 9: Diseño del triplete de Cooke realizado para $u_{1}=h / l$

representación grafica del recorrido de los rayos atraves del sistema óptico, Figura 10 diferentes ventanas brindan información del ángulo refractado, la altura de la imagen en cada superficie y las diferentes longitudes focales se resumen en la Tabla 5 .

La Tabla 5 y la Figura 10 evalúa y grafica las condiciones correspondientes a la Tabla 1, como datos de entrada, para el uso del programa OSLO EDU con $u_{1}=0$. Comparando las Tablas 5 y 2 , las diferencias de $u_{1}$ y $h$ son del orden de centésimas y décimas de milimetro respectivamente.

Tabla 5: Ángulo refractado y tamaño de la imagen para cada superficie para $u_{1}=0$ con el programa OSLO EDU.

\begin{tabular}{lcc}
\hline Superficie & $u_{i}^{\prime}$ & $h_{i}(\mathrm{~mm})$ \\
\hline 1 & $-0,15822$ & 16,700 \\
2 & $-0,27313$ & 15,751 \\
3 & $-0,06239$ & 13,019 \\
4 & 0,09998 & 12,957 \\
5 & 0,03927 & 14,037 \\
6 & $-0,16774$ & 14,273 \\
\hline
\end{tabular}

Longitud focal efectiva $e f l=99,7562 \mathrm{~mm}$

Longitud focal posterior $b f l=85,2558 \mathrm{~mm}$ Amplificación transversal $m=0,7660$

Posición del plano principal $\delta=-14,5004 \mathrm{~mm}$

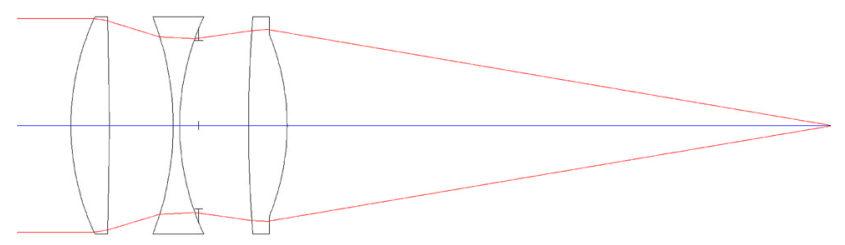

Figura 10: Triplete de Cooke para $u_{1}=0$ realizado con el programa OSLO EDU. 
La simulación realizada por el código presentado en este trabajo, muestra mínimas diferencias con el realizado por el programa OSLO EDU, lo que significa que el diseño del triplete de Cooke elaborado con el código minimiza las aberraciones. El código se anexa en el apéndice del trabajo.

\section{Conclusiones}

Ante la carencia de experimentos de laboratorios en la enseñanza del curso de óptica, una alternativa es el desarrollo de simples programas que pueden realizar simulaciones relacionadas a la geometría óptica.

El código realizado en el programa MATLAB consiguío modelar y simular el triplete de Cooke con su característica más importante; anular la presencia de aberraciones. El comportamiento y cálculo de la refracción de los haces para las seis superficies se comparó con el desarrollado por el programa OSLO EDU y se aprecia en ambos casos valores que difieren en el orden de las milésimas. Tambien el tamaño y la ubicación de la imagen final pudieron ser determinadas y calculadas. Asi como la versión educativa del OSLO EDU es usada para el estudio de la óptica básica y el diseño de algunos instrumentos básicos que permiten visualizar el detalle de la aplicación de los modelos de la óptica geométrica y paraxial, los estudiantes pueden con simples códigos recrear, simular y mejorar su comprensión de los procesos de la formación de imágenes en los diferentes dispositivos ópticos.

Lo más importante en la elaboración de este código es que se puede aplicar a cualquier superficie sin limite de cantidad; incrementando rayos paraxiales, marginales y elementos estandares (lentes y espejos). Un futuro trabajo es el desarrollo a partir de un GUI [9], de un banco óptico virtual con diferentes e ilimitados elementos reflexivos y refractivos donde se puede realizar simulaciones relacionadas con la propagación de la luz y la formación de imágenes ópticas semejantes a una actividad experimental real; esto representa una ventaja frente al programa OSLO EDU, que por su condición de gratuidad solo procesa hasta un máximo de diez superficies.

\section{Material suplementario}

El siguiente material suplementario está disponible en línea:

Apéndice

\section{Referencias}

[1] OSLO EDU, disponible en https://www.lambdares. com/oslo-usonly/, accesado en 5/6/2019.

[2] https://www.synopsys.com/optical-solutions/ codev/optical-design.html

[3] https://www.zemax.com/products/opticstudio

[4] L. Seidel, Ueber die Theorie der Fehler, mit welchen die durch optische Instrumente gesehenen Bilder, behaftet sind, und über die mathematischen Bedingungen ihrer Aufhebung (Bayerischen Akademie der Wissenschaften, München, 1857) p. 227.

[5] www.schott.com/advanced_optics/abbe_datasheet/ schott-datasheet-n-sk4.pdf

[6] www.advancedglass.net/pdfdocs/Optical\%20Glass\% 20Inventory.pdf

[7] W.J. Smith, Modern Optical Engineering (Mac Graw Hill, New York, 2008) p. 22.

[8] https://la.mathworks.com/products/matlab.html

[9] https://la.mathworks.com/discovery/matlab-gui. html 